\title{
A Brain-Machine Interface using Dry-Contact, Low-Noise EEG Sensors
}

\author{
Thomas J. Sullivan, Stephen R. Deiss \\ Division of Biological Sciences \\ UC San Diego \\ La Jolla, CA
}

Email: tom@sullivan.to, sdeiss@ucsd.edu

\author{
Tzyy-Ping Jung \\ Inst. for Neural Computation, UCSD \\ and National Chiao Tung University \\ Hsinchu, Taiwan, ROC \\ Email: jung@sccn.ucsd.edu
}

\author{
Gert Cauwenberghs \\ Division of Biological Sciences \\ UC San Diego \\ La Jolla, CA \\ Email: gert@ucsd.edu
}

\begin{abstract}
Electroencephalograph (EEG) recording systems offer a versatile, non-invasive window on the brain's spatiotemporal activity for many neuroscience and clinical applications. Our research aims to improve the convenience and mobility of EEG recording by eliminating the need for conductive gel and creating sensors that fit into a scalable array architecture. The EEG drycontact electrodes are created with micro-electrical-mechanical system (MEMS) technology. Each channel of our analog signal processing front-end comes on a custom-built, dime-sized circuit board which contains an amplifier, filters, and analog-to-digital conversion. A daisy-chain configuration between boards with bitserial output reduces the wiring needed.

A system consisting of seven sensors is demonstrated in a realworld setting. Consuming just $3 \mathrm{~mW}$, it is suitable for mobile applications. The system achieves an input-referred noise of 0.28 $\mu$ Vrms in the signal band of 1 to $100 \mathrm{~Hz}$, comparable to the best medical-grade systems in use. Noise behavior across the daisychain is characterized, alpha-band rhythms are detected, and an eye-blink study is demonstrated.
\end{abstract}

\section{INTRODUCTION}

Electroencephalograph (EEG) systems record electrical potentials from various locations on the scalp. These potentials are generated by neural activity within the brain and may ultimately shed light on the its inner workings. The EEG electrodes are typically made of metal and make contact with the skin through an electrically-conductive gel. They are mounted on a cap that the subject wears on the head. These systems have many research and clinical applications ([2], for example).

Two issues limit the use of these systems. The first is that electrically-conductive gel is required for a good connection between the sensors and the scalp. This gel takes a lot of time to apply, it limits the realizable density of sensors on the scalp, and it tends to dry out, which limits the recording time. Here, we use electrodes that were created in a MEMS technology and do not require gel [1]. We follow this by a circuit that processes the signal. The second limiting issue with typical EEG systems is that they are not mobile. They have high power consumption and a mass of wires connecting the cap to a base station that processes the signal and records them. Our sevenelectrode system (which can be easily expanded) consumes only $3 \mathrm{~mW}$ of power making battery power feasible. Also, in our system the number of wires needed to interface with a large number of electrodes is reduced since the power, clocks, and measured signals are daisy-chained from one board to another. The system is also easily adapted to work with a wireless module.

In this work, we report on experiments performed with our system containing seven non-contact sensors (characterization of an individual sensor was previously reported [3]). The experiments take place in an electrical engineering laboratory, not the more typical Faraday cage. The magnitude of sixty hertz noise and influence of reference location within the daisy-chain are reported. Additionally, data from two EEG experiments show the successful collection of useful EEG data in this environment.

\section{SENSOR DESIGN}

The analog front-end circuit is shown in Figure 1. It consists entirely of off-the-shelf components, which keeps the overall system cost low. The circuit amplifies the incoming EEG signal, filters out frequencies that are not in the band of interest, and digitizes the signal. The gain over frequency of this circuit was previously reported [3], as was the noise level of $0.28 \mu \mathrm{Vrms}$ and power consumption of $423 \mu \mathrm{W}$.

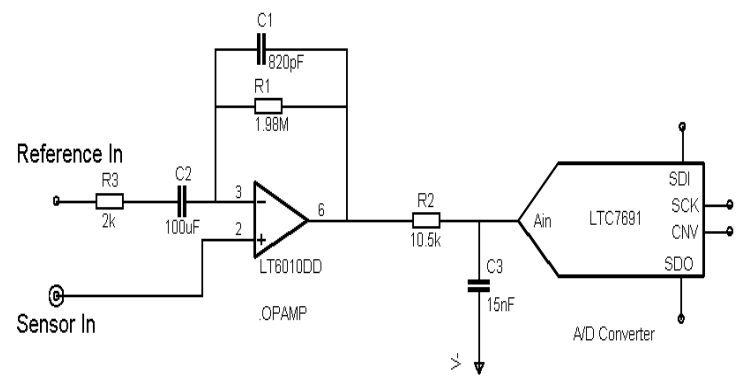

Fig. 1. Analog Front-end Schematic. The circuit consists of an off-the-shelf amplifier and analog-to-digital converter. The mid-band gain is 1000 with noise filtered out below $1 \mathrm{~Hz}$ and above $100 \mathrm{~Hz}$.

The low-noise LT6010 operational amplifier is configured for a mid-band gain of 1000 . A $100 \mu \mathrm{F}$ capacitor is used to cut the gain to 1 at frequencies below $1 \mathrm{~Hz}$. This is important since the sensor/scalp interface often generates significant DC offset voltages up to $25 \mathrm{mV}$. A capacitor in the feedback path, $\mathrm{C} 1$, creates a low-pass filter with a $-3 \mathrm{~dB}$ cutoff at $100 \mathrm{~Hz}$. 

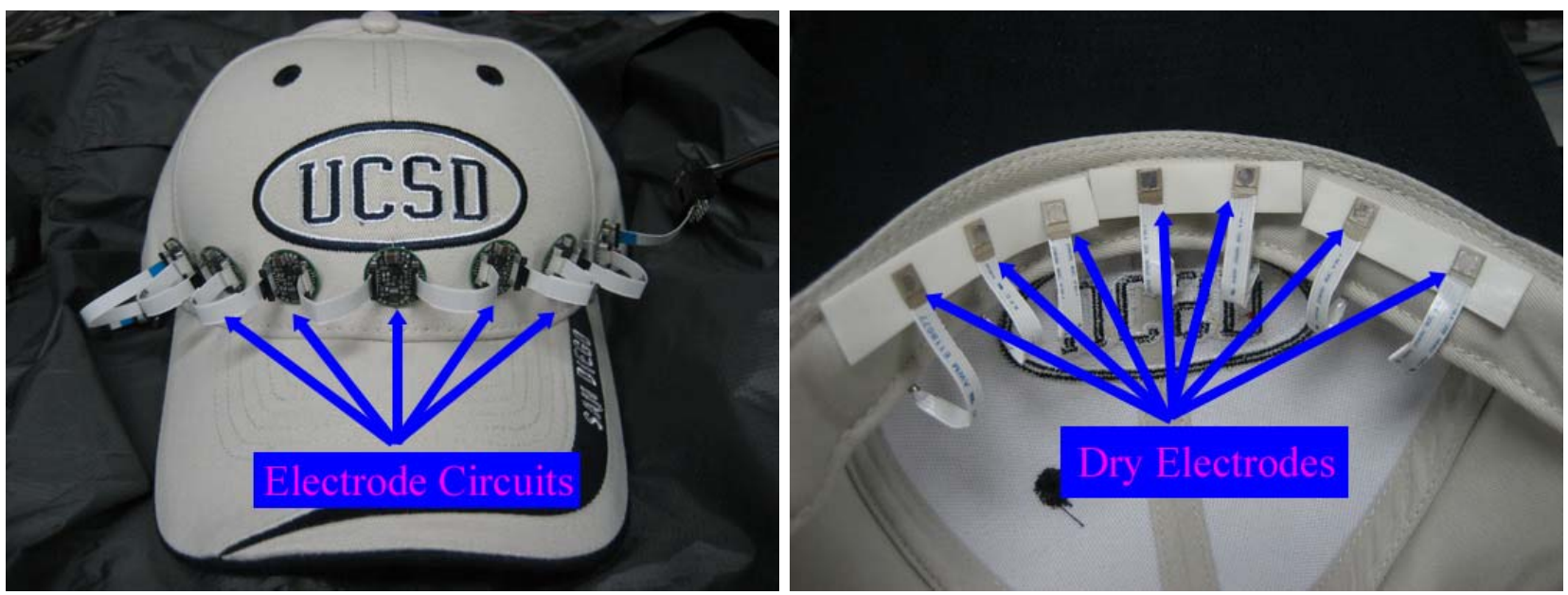

Fig. 2. Photographs of EEG system. (Left) Seven electrode circuits are mounted onto a baseball cap. This is not a traditional approach to building EEG recording devices, but may be feasible with the latest dry sensors. (Right) The dry sensors are mounted onto the inside brim of the baseball cap to get EEG readings from the forehead of the subject.

A second pole is set up at $1 \mathrm{kHz}$ by $\mathrm{R} 2$ and $\mathrm{C} 3$. After the filtering the voltage is sampled at $2 \mathrm{kS} / \mathrm{s}$ by the LTC7691 analog-to-digital converter (ADC), which has 18-bit resolution and consumes only $20 \mu \mathrm{A}$. While EEG signals generally do not extend beyond $100 \mathrm{~Hz}$, noise from muscle movements is often found in frequencies up to $1 \mathrm{kHz}$. The signals in this band are digitized because this muscle noise may be a valuable signal for researchers doing EEG experiments in which the subjects are moving around and interacting with the world.

The circuit was built on a custom PCB (seen in Figure 2 (left panel). The circuit is roughly the size of a dime. The signal is connected to the sensor with a pin extending from the bottom of the board in the center. The two ICs are in MSOP and DFN packages. The passive components are all in 0603 packages (measuring 60 mils by 30 mils) except for the large $100 \mu \mathrm{F}$ capacitor in a 1210 package. The two connectors on either side of the boards connect to other EEG sensor circuit boards and serve two purposes: (1) to share the power, reference voltage, and ADC clocks, and (2) to daisy chain the digital outputs. Configuring boards into a daisy chain means that the output of one circuit will be passed to another board. All the outputs are, in effect, concatenated into one long serial bit-stream and read by the data acquisition system. When many electrodes are used on one EEG cap (256, for example) passing all these signals from one board to another drastically reduces the number of wires that are required for control and read-out.

The signal is obtained from the dry electrode reported elsewhere [1]. They are located on the inside brim of the baseball cap as shown in Fig. 2 (right panel). These pieces of coated silicon, measuring $4 \mathrm{~mm}$ on a side, are made using a MEMS process. The microscopic needles that jut out from the silicon plane increase the surface area of the electrode and achieve a lower contact resistance than typical dry electrodes.

\section{EXPERIMENTS}

Often, EEG experiments are performed in a Faraday cage that significantly reduces electromagnetic interference from outside sources. This is not practical for many situations and thankfully is not always required in order to get useful data. Several experiments were performed in an unaltered electrical engineering laboratory in order to measure the noise produced and the type of useful data that can be obtained.

\section{A. Noise Characterization}

Fig. 3 shows the power of the noise around $60 \mathrm{~Hz}$. In this seven sensors were employed in the system. Sensor 7 was placed behind one ear and used as a reference. This is a typical reference position in EEG experiments since little EEG signal can be detected there. The time-varying voltages of each of the remaining sensors were subtracted from the reference in order to cancel some of the common noise picked up from the environment. From the figure, it is apparent that a strong trend exists in that the sensors further from the reference record more $60 \mathrm{~Hz}$ noise (as well, presumably, as noise at other frequencies). This is evidence that the noise picked-up from the environment is unevenly distributed across the scalp and shows that more noise can be canceled when physically closer to the reference. The noisiest sensor reports a $60 \mathrm{~Hz}$ power level roughly ten times the useful $10 \mathrm{~Hz}$ signal reported in experiments below.

In order to test the effects of reference location further another experiment was performed. The sensor inputs from 8 sensors were all connected to the same reference voltage on a PCB. Data was recorded from the outputs for 30 seconds. A notch filter at $60 \mathrm{~Hz}$ was employed in software after the data was collected in order to eliminate the dominant line noise source. Fig. 4 shows the measured noise from each sensor integrated over the $1-100 \mathrm{~Hz}$ frequency band. The calculations were performed three different times, each time with a different sensor used as the reference (Sensor 1, 4, or 


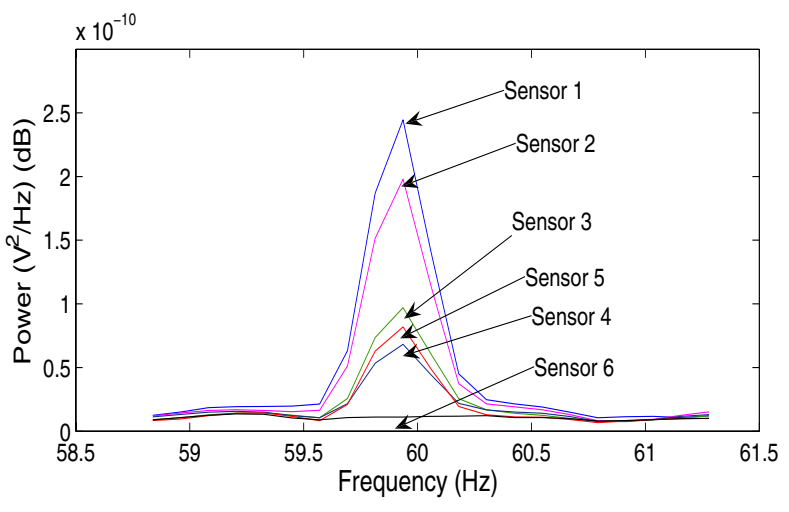

Fig. 3. Power of $60 \mathrm{~Hz}$ noise. Sensor 7 was placed behind the ear and used as a reference. The power in the frequency range of line noise is shown for the other 6 sensors. The sensors closer to the reference have lower noise levels.

8). It is obvious once again that lower noise is obtained when closer to the reference sensor. The measured noise when near the reference compare favorably to the $0.28 \mathrm{uVrms}$ reported previously using a one-sensor system [3].

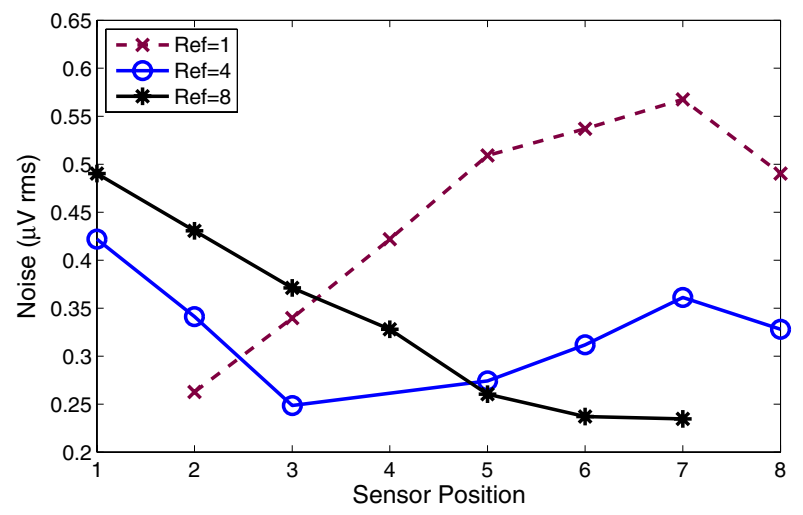

Fig. 4. Effect of reference position. Noise was recorded with the sensor inputs were connected to a reference voltage on a PCB. The noise from each sensor integrated over the $1-100 \mathrm{~Hz}$ band is shown. The three lines correspond to the use of three different sensors as the reference. Sensors closer to the reference record lower noise.

\section{B. EEG Data}

A test was performed in which EEG data was taken from a human subject. In one test, the subject started with his eyes closed for a 15 second block of time before keeping his eyes open for 10 seconds. The power spectral density of a voltage recorded by one of the electrodes is shown for both blocks of time. With the eyes closed there is a marked increase in power around 9 Hertz. This increase in what is called the alpha band is typical when eyes are closed. A normalized spectrogram of the whole 25 seconds is also shown in Fig. 5. The decrease in the alpha band, as well as a slight increase in higher frequencies, is noticeable around the 15 second mark when the subject's eyes were opened. The power spectral densities of the two blocks of time are shown in Fig. 6. This is a typical
EEG signal which is currently being investigated by the brain research community.

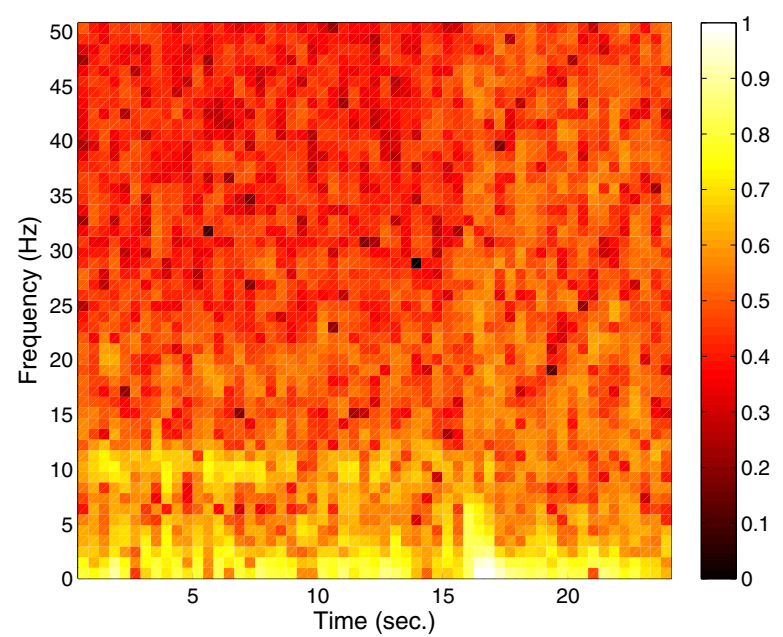

Fig. 5. Spectrogram of Closed Eyes Experiment. The power in the $8-13 \mathrm{~Hz}$. alpha band decreases when the eyes are opened at around 15 seconds. The higher frequencies appear to increase slightly.

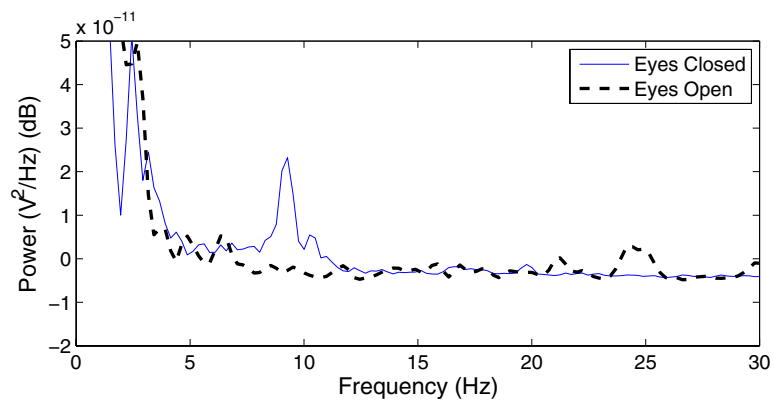

Fig. 6. Alpha Waves. The power spectral density of two time blocks are shown. During one period the subject kept his eyes open, during the other his eyes were closed. The characteristic bump at $10 \mathrm{~Hz}$ is a result of $\alpha$-waves that typically appear when subjects close their eyes.

In another test, the subject was asked to blink his eyes in a specific pattern. Four typical blinks of both eyes were followed by alternating between blinking left and right eyes. The resulting EEG patterns of 6 EEG channels are shown in Figure 7. The first four deflections in the EEG voltages are typical of eye blinks. The voltages following this are different for the six electrode locations. The difference between the voltages from the left-most and right-most electrodes are shown in Figure 8. It is obvious from this plot when a lefteye blink was performed (large upward voltage deflection) and when a right-eye blink was performed (large downward deflection).

\section{DISCUSSION}

Experiments using a new EEG system are described here. Noise across a daisy-chain of sensors is shown to depend on the sensor location relative to the reference. Low-noise 

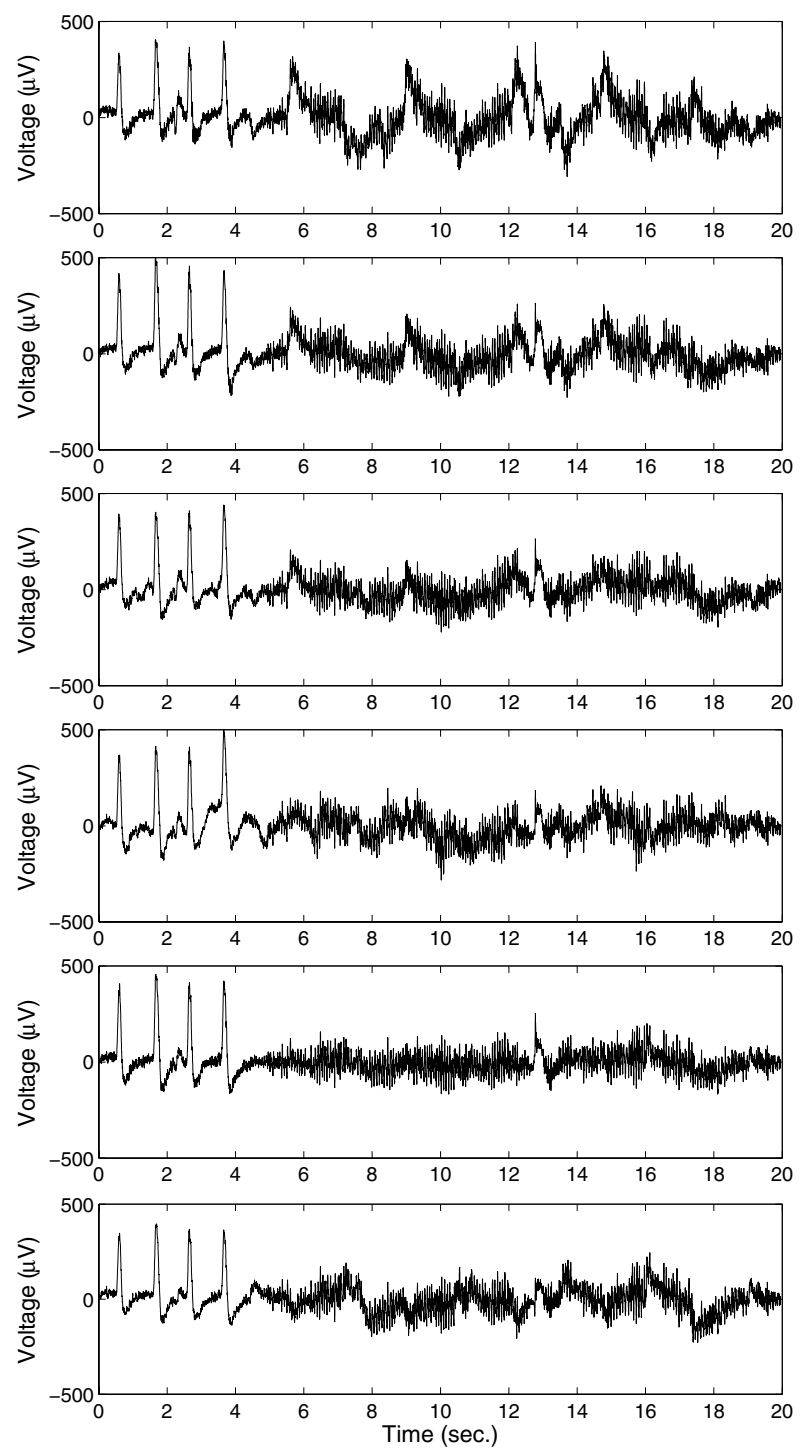

Fig. 7. Blinking Experiment. Sensor data from six locations is shown from an experiment. The subject blinked his eyes regularly 4 times, then alternated between blinking his left and right eyes.

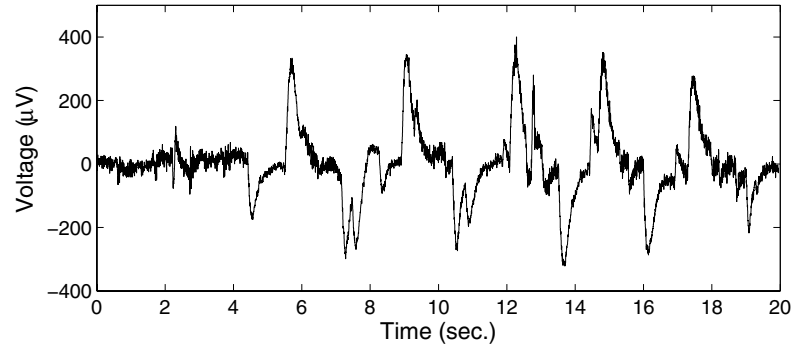

Fig. 8. Left/Right Blinks. The difference between the output voltages from the left-most and right-most sensors is shown. Upward voltage deflections correspond to a left-eye blink, while right-eye deflections correspond to a right-eye blink. When both eyes blink, the sensor voltages are roughly the same and cancel each other out. operation is achieved, comparable to current medical-grade systems. Two experiments were described that show robust EEG data collection even in a noisy lab environment. First, alpha waves around $10 \mathrm{~Hz}$ were seen to increase in power with closed eyes, which is a commonly reported finding. Second, eye blinks from left or right eyes were detected with electrodes place on the forehead.

The sensors used in the system use MEMS-based, dry electrodes that create a good contact with the skin without requiring gel. This makes for a much more convenient experiment setup and enables experiments requiring long-term recording. An additional feature of our front-end circuitry is that it can be used equally well with dry sensors or gel-based sensors. We address the need for mobility in EEG recording equipment by creating a daisy-chain of digital outputs consisting of all the electrode boards. This way, the wiring requirements for power, control, and read-out are reduced making a wireless solution more feasible. Also, mobility is possible because only a small amount of power is required for the whole signal processing system. Small batteries can be employed to power the system for an extended period of time.

Future work could extend the system to a larger number of sensors and demonstrate long-term recording. We would also like to take the system wireless by adding a small module to the cap. Additionally, we would like to investigate the requirement of contact with the skin [4].

\section{ACKNOWLEDGEMENTS}

The authors would like to thank Ruey-Song Huang for help in the testing of this circuit and Scott Makeig for stimulating discussions. We would like to thank Dr. C.T. Lin, Dr. J.C. Chiou, and the members of the National Chiao Tung University Brain Research Center for use of their dry MEMS sensors. This work was supported by DARPA.

\section{REFERENCES}

[1] J. C. Chiou, Li-Wei Ko, Chin-Teng Lin, Chao-Ting Hong, Tzyy-Ping Jung, "Using Novel MEMS EEG Sensors in Detecting Drowsiness Application," IEEE Biomedical Circuits and Systems Conference, 2006.

[2] T. P. Jung, S. Makeig, M. Stensmo, and T. Sejnowski, "Estimating alertness from the EEG power spectrum," IEEE Transactions on Biomedical Engineering 44:60-69, 1997.

[3] T. Sullivan, S. Deiss, T.P. Jung, and G. Cauwenberghs, "A Low-Noise, Low-Power EEG Acquisition Node for Scalable Brain-Machine Interfaces", In Proceedings of the SPIE Conference on Bioengineered and Bioinspired Systems III, May 2-4 2007.

[4] T. Sullivan, S. Deiss, and G. Cauwenberghs, "A Low-Noise, Non-Contact EEG/ECG Sensor”, IEEE Biomedical Circuits and Systems Conf., 2007. 